\title{
Implementation Insights
}

\author{
Kurt C. Stange, MD, PbD, Editor \\ Ann Fam Med 2009;7:179-181. DOI: 10.1370/afm.966.
}

M uch of the Annals online discussion during the past 2 months addresses how scientific information can be implemented in real-world practice, communities, and systems.

There is a larger context for this discussion. Recent research program announcements from the US National Institutes of Health distinguish between dissemination and implementation. ${ }^{1}$ Dissemination is the targeted distribution of information and intervention materials to a specific public health or clinical practice audience. The intent is to spread knowledge and the associated evidence-based interventions. Implementation is the use of strategies to introduce or change evidence-based health interventions within specific settings.

In January, the oversubscribed 2nd Annual NIH Conference on the Science of Dissemination and Implementation: Building Research Capacity to Bridge the Gap From Science to Service (http://conferences.thehillgroup.com/obssr/di2008/about.html) provided a forum for researchers in this emerging and important field.

Importantly, the Annals discussion represents both the generators and users of implementation knowledge and provides frontline experience. Discussants raise the following observations and hypotheses about effective implementation:

- Asthma medication prescription ${ }^{2}$ may be less likely among physicians with greater experience of the benign course of much of the bronchospastic disease seen among children in primary care. ${ }^{3}$

- A reductionist approach that emphasizes biologically observable events, ${ }^{4}$ while useful, is an impoverished framework for understanding spirituality. ${ }^{5}$

- The phenomenon of healing ${ }^{6}$ may have much in common with identity development. ${ }^{7}$

- Understanding of chronic fatigue ${ }^{8}$ is enhanced by clustering patients into patterns. Earlier recognition of chronic fatigue syndrome may be important because of the helpfulness of early treatment. ${ }^{9}$

- "The providers are not the only element in the implementation process ${ }^{10} ;$ the interaction of the practices' culture, leadership, prior change history, and any external influences need consideration." ${ }^{11}$

- An alternative to trying to find "the needle in a haystack" of the suicidal adolescent ${ }^{12}$ is to help depressed adolescents develop protective factors to diminish the likelihood of suicide. ${ }^{13}$

- The utility of a screening tool for impaired glucose tolerance ${ }^{14}$ would be helped by validated thresholds for useful action. ${ }^{15,16}$

- Colorectal cancer testing ${ }^{17-19}$ is enhanced by:

- "[T]he interplay between health insurance and having a personal physician" 20

- "Team care and tracking systems" 21

- Use of a simple, easy-to-perform noninvasive test with adequate training and explanation among motivated patients who were coming in anyway for flu shots. ${ }^{22}$

- "[A]n intervention that did not require a primary care clinic visit," plus electronic medical records that identified eligible patients in advance of a flu shot clinic visit, sufficient nonphysician personnel, and resources that assured patients with positive tests had access to follow-up diagnostic evaluations. ${ }^{23}$

- Multiple strategies, including "(1) promotion by clinicians in the context of an office visit ${ }_{i}$ (2) promotion by non-physician staff who interact with patients before or after doctor visits, (3) routine mailing of kits to patients who are due; and (4) at other times when patients come into contact with the medical system, such as when they are coming in for an annual flu shot."24

- Treating colonoscopy as a skill-based ${ }^{25-27}$ or as a specialty-training-based ${ }^{28,29}$ procedure. Primary care endoscopists may be a solution to projected endoscopist shortages. ${ }^{30}$

- Delivery of colonoscopy services in light of multiple factors, including markers of technical proficiency, team approaches with diverse disciplines, local medical resources, and the supply of endoscopists compared with the needs of the population. ${ }^{25-32}$

Direct-to-consumer (DTC) advertising of medications $^{33}$ requires time in the patient visit that "may lead to further displacement of medically important services." ${ }^{134}$ Although it can be used to build teachable moments, ${ }^{35,36}$ it leads to prescription of the requested drug the majority of the time, even though physicians 
deny feeling pressured to prescribe. ${ }^{37}$ It is wrong and should be banned. ${ }^{38}$

Regarding intimate partner violence involving male perpetrator/victims, ${ }^{39}$ a short screening tool and a brief intervention may increase the likelihood of it being dealt with. ${ }^{40} \mathrm{~A}$ new paradigm is emerging for a more integrated response than treating victims medically and perpetrators in the criminal justice setting by using the acute health care setting to identify and treat the many perpetrators who also are victims. ${ }^{41}$

Screening for postpartum depression ${ }^{42}$ is important, and requires both screening and follow-up, ${ }^{43-47}$ and it may be enhanced by a 2-step method in which the first step involves a 2 -item screen, since this breaks down the time barrier. ${ }^{48,49}$

Regarding postmyocardial (post-MI) infarction depression, ${ }^{50}$ family physicians are ideally positioned to integrate care, ${ }^{51,52}$ and post-MI depression may be reduced by cardiac rehabilitation exercise. ${ }^{53}$

Reducing racial disparities in hypertension control $^{54,55}$ is complicated, ${ }^{56}$ may affect blacks in different countries differently, ${ }^{57}$ and needs to recognize that "rising waters for all may not necessarily lift all boats equally." That is, special efforts may be needed for certain groups, rather than assuming that quality improvement efforts will improve care for all..$^{58}$

Please join the discussion at http://www. AnnFamMed.org.

\section{References}

1. NIH Program Announcements: PAR-06-039, PAR-07-086, PAR-06520. Dissemination and Implementation Research in Health. http:// grants.nih.gov/grants/guide/pa-files/PAR-06-039.html, Accessed Jan 30, 2009.

2. Zuidgeest MG, van Dijk L, Spreeuwenberg P, et al. What drives prescribing of asthma medication to children? A multilevel population-based study. Ann Fam Med. 2009;7(1):32-40.

3. van der Wouden JC. How to explain these findings [eletter]? http:// www.annfammed.org/cgi/eletters/7/1/32\#10558, 29 Jan 2009.

4. Davidson RJ. Spirituality and medicine: science and practice. Ann Fam Med. 2008;6(5):388-389.

5. Daaleman TP. An impoverished view of spirituality [eletter]. http:// www.annfammed.org/cgi/eletters/6/5/388\#10415, 26 Dec 2008.

6. Meza JP, Fahoome GF. The development of an instrument for measuring healing. Ann Fam Med. 2008;6(4):355-360.

7. Bibace $R$, et al. "Healing"-A phenomenon with many different names [eletter]. http://www.annfammed.org/cgi/eletters/6/4/355\#10522, 23 Jan 2009.

8. Nijrolder I, van der Windt DA, van der Horst HE. Prognosis of fatigue and functioning in primary care: a 1-year follow-up study. Ann Fam Med. 2008;6(6):519-527.

9. Wilborg JF, et al. Patients with CFS are under-diagnosed by general practitioners [eletter]. http://www.annfammed.org/cgi/eletters/6/6/519\#10404, 12 Dec 2008.

10. Hahn KA, Ferrante JM, Crosson JC, Hudson SV, Crabtree BF. Diabetes flow sheet use associated with guideline adherence. Ann Fam Med. 2008;6(3):235-238.
11. Homa K. What do we consider an exposure to an evidencedbased tool use [eletter]? http://www.annfammed.org/cgi/eletters/6/3/235\#10419, 7 Jan 2009.

12. Van Voorhees BW, Paunesku D, Gollan J, Kuwabara S, Reinecke M, Basu A. Predicting future risk of depressive episode in adolescents: the Chicago Adolescent Depression Risk Assessment (CADRA). Ann Fam Med. 2008;6(6):503-511.

13. Bell CC.. Wonderful example of modern technology designed to prevent psychiatric illness [eletter]. http://www.annfammed.org/cgi/ eletters/6/6/503\#10400, 10 Dec 2008.

14. Koopman RJ, Mainous III AG, Everett CJ, Carter RE. Tool to Assess Likelihood of Fasting Glucose ImpairmenT (TAG-IT). Ann Fam Med. 2008;6(6):555-561.

15. Cummings DM. Detecting impaired fasting glucose [eletter]. http:// www.annfammed.org/cgi/eletters/6/6/555\#10417, 5 Jan 2009.

16. Franciosi M. Prediabetes and screening tools [eletter]. http://www. annfammed.org/cgi/eletters/6/6/555\#10393, 8 Dec 2008.

17. Cardarelli R, Thomas JE. Having a personal health care provider and receipt of colorectal cancer testing. Ann Fam Med. 2009;7(1):5-10.

18. Potter MB, Phengrasamy L, Hudes ES, McPhee SJ, Walsh JM. Offering annual fecal occult blood tests during flu shot clinics increases colorectal cancer screening rates. Ann Fam Med. 2009;7(1):17-23.

19. Wilkins T, LeClair B, Smolkin M, et al. Screening colonoscopies by primary care physicians: a meta-analysis. Ann Fam Med. 2009;7(1):56-62.

20. Roetzheim R. Health insurance versus personal physician [eletter]. http://www.annfammed.org/cgi/eletters/7/1/5\#10478, 16 Jan 2009.

21. Rosenthal TC. Independent corroboration [eletter]. http://www. annfammed.org/cgi/eletters/7/1/5\#10435, 15 Jan 2009.

22. Levin TR. Increasing CRC screening by employing ancillary staff [eletter]. http://www.annfammed.org/cgi/eletters/7/1/17\#10449, 15 Jan 2009.

23. Hoffman RM. Thinking outside of the clinic visit box [eletter]. http:// www.annfammed.org/cgi/eletters/7/1/17\#10555, 29 Jan 2009.

24. Potter MB. Multiple strategies needed to maximize screening rates [eletter]. http://www.annfammed.org/cgi/eletters/7/1/17\#10475, 16 Jan 2009.

25. Wilkins T, et al. Response to Dr Rex's comments [eletter]. http:// www.annfammed.org/cgi/eletters/7/1/56\#10551, 28 Jan 2009.

26. Kolber M. Primary care endoscopy [eletter]. http://www. annfammed.org/cgi/eletters/7/1/56\#10509, 20 Jan 2009.

27. Potter MB. Primary care colonoscopy can be high quality colonoscopy [eletter]. http://www.annfammed.org/cgi/eletters/7/1/56\#10430, 15 Jan 2009.

28. Rex DK. Quality colonoscopy [eletter]. http://www.annfammed.org/ cgi/eletters/7/1/56\#10480, 19 Jan 2009.

29. Foxx-Orenstein AE. The quality of the examination is key [eletter]. http://www.annfammed.org/cgi/eletters/7/1/56\#10444, 15 Jan 2009.

30. Wilkins $T$, et al. Re: the quality of the examination is key [eletter]. http://www.annfammed.org/cgi/eletters/7/1/56\#10464, 15 Jan 2009.

31. Shah SA. Invited response to Wilkins study in Annals of Family Medicine [eletter]. http://www.annfammed.org/cgi/eletters/7/1/56\#10567, 3 Feb 2009.

32. Wilkins T. Re: Primary care colonoscopy can be high quality qolonoscopy [eletter]. http://www.annfammed.org/cgi/eletters/7/1/56\#10466, 15 Jan 2009.

33. Parnes B, Smith PC, Gilroy C, et al. Lack of impact of direct-to-consumer advertising on the physician-patient encounter in primary care: A SNOCAP report. Ann Fam Med. 2009;7(1):41-46.

34. Cronholm PF, et al. The health service burden of "low-impact" Direct-to-Consumer advertising [eletter]. http://www.annfammed. org/cgi/eletters/7/1/41\#10517, 22 Jan 2009.

35. Parnes B. Response to Drs Allen and Brody [eletter]. http://www. annfammed.org/cgi/eletters/7/1/41\#10471, 15 Jan 2009. 
36. Allen RE. DTCA helpful, but not the key. So what does cause people to seek care [eletter]? http://www.annfammed.org/cgi/eletters/7/1/41\#10433, 15 Jan 2009.

37. Brody H. So why does DTCA stir us up so much [eletter]? http:// www.annfammed.org/cgi/eletters/7/1/41\#10437, 15 Jan 2009.

38. Loveless HR. Ban the ads [eletter]. http://www.annfammed.org/cgi/ eletters/5/2/180\#10488, 19 Jan 2009.

39. Rhodes KV, Houry D, Cerulli C, Straus H, Kaslow NJ, McNutt L. Intimate partner violence and comorbid mental health conditions among urban male patients. Ann Fam Med. 2009;7(1):47-55.

40. Ernst AE. Screening males for IPV perpetration and victimization [eletter]. http://www.annfammed.org/cgi/eletters/7/1/47\#10511, 20 Jan 2009.

41. Cronholm PF, et al. Health service collaborations in the engagement of intimate partner violence perpetration [eletter]. http:// www.annfammed.org/cgi/eletters/7/1/47\#10494, 20 Jan 2009.

42. Gjerdingen D, Crow S, McGovern P, Miner M, Center B. Postpartum depression screening at well-child visits: validity of a 2-question screen and the PHQ-9. Ann Fam Med. 2009;7(1):63-70.

43. Buist AE. Importance of screening [eletter]. http://www.annfammed. org/cgi/eletters/7/1/63\#10500, 20 Jan 2009.

44. Gjerdingen DK. Postpartum depression screening at well-child visits: validity of a 2-question screen and the PHQ-9 [eletter]. http:// www.annfammed.org/cgi/eletters/7/1/63\#10526, 25 Jan 2009.

45. Stewart DE. Postpartum depression screening [eletter]. http://www. annfammed.org/cgi/eletters/7/1/63\#10496, 20 Jan 2009.

46. McRoberts SA. Time well spent [eletter]. http://www.annfammed. org/cgi/eletters/7/1/63\#10490, 19 Jan 2009.
47. Stenhjem P. I wish screening had been available for me [eletter] http://www.annfammed.org/cgi/eletters/7/1/63\#10485, 19 Jan 2009.

48. Seehusen DA. Breaking down the time barrier [eletter]. http://www. annfammed.org/cgi/eletters/7/1/63\#10544, 27 Jan 2009.

49. Boyd RC. Screening for postpartum depression with the patient health questionnaire (PHQ): a commentary [eletter]. http://www. annfammed.org/cgi/eletters/7/1/63\#10535, 25 Jan 2009.

50. Green LA, Dickinson W, Nease DE, et al. AAFP guideline for the detection and management of post-myocardial infarction depression. Ann Fam Med. 2009;7(1):71-79.

51. Green LA. Re: Cardiac rehab in post-MI depression [eletter]. http:// www.annfammed.org/cgi/eletters/7/1/71\#10524, 25 Jan 2009.

52. Erwin JP. Cardiac rehab in post-MI depression [eletter]. http://www. annfammed.org/cgi/eletters/7/1/71\#10442, 15 Jan 2009.

53. Schwenk TL. Why family physicians are important [eletter]. http:// www.annfammed.org/cgi/eletters/7/1/71\#10446, 15 Jan 2009.

54. Fiscella K, Holt K. Racial disparity in hypertension control: tallying the death toll. Ann Fam Med. 2008;6(6):497-502.

55. Satcher D. Examining racial and ethnic disparities in health and hypertension control. Ann Fam Med. 2008;6(6):483-485.

56. Scanlan J. Measuring racial disparities in hypertension control [eletter]. http://www.annfammed.org/cgi/eletters/6/6/483\#10530, 25 Jan 2009.

57. Onuigbo MN. HTN, racial disparity and result [eletter]. http://www. annfammed.org/cgi/eletters/6/6/497\#10421, 13 Jan 2009.

59. Fiscella K. Thoughtful comments [eletter]. http://www.annfammed. org/cgi/eletters/6/6/497\#10423, 15 Jan 2009.

\section{CORRECTION}

Ann Fam Med 2009;7:181. DOI: 10.1370/afm.984.

The January/February 2009 article by Thad Wilkins and colleagues (Wilkins T, LeClair B, Smolkin M, et al. Screening colonoscopies by primary care physicians: a meta-analysis. Ann Fam Med. 2009;7(1):56-62), had an incorrect entry in Table 2, under Estimated Adenocarcinoma Detection Rate (95\% CI) for Lloyd, 2006. This entry should read: 0.5 (0.46-0.54). The corrected table is shown below.

Table 2. Estimated Rates of Colonoscopy Quality Outcomes Using Random Effects Modeling

\begin{tabular}{|c|c|c|c|c|c|}
\hline Study, Year & $\begin{array}{c}\text { Estimated } \\
\text { Adenoma Detection } \\
\text { Rate }(95 \% \mathrm{Cl})\end{array}$ & $\begin{array}{c}\text { Estimated } \\
\text { Adenocarcinoma } \\
\text { Detection Rate } \\
(95 \% \mathrm{Cl})\end{array}$ & $\begin{array}{c}\text { Estimated } \\
\text { Reach-the-Cecum } \\
\text { Rate (All Patients) } \\
(95 \% \mathrm{Cl})\end{array}$ & Perforations $^{a}$ & $\begin{array}{c}\text { Bleeding } \\
\text { Not } \\
\text { Requiring } \\
\text { Transfusion }^{\mathrm{a}}\end{array}$ \\
\hline Godreau, ${ }^{24} 1992$ & $51.7(44.0-59.3)$ & $2.2(0.9-5.7)$ & $83.2(76.6-88.2)$ & 0 & 0 \\
\hline Rodney et al, ${ }^{25} 1993$ & $8.8(6.1-12.5)$ & $2.0(0.9-4.2)$ & 48.7 (43.1- 54.4) & 0 & 0 \\
\hline Hopper et al, ${ }^{26} 1996$ & $43.7(40.7-46.7)$ & Not reported & $74.8(72.1-77.3)$ & 0 & 0 \\
\hline Harper et al27, 1997 & $20.9(16.8-25.8)$ & $3.5(2.0-6.1)$ & $87.1(82.9-90.4)$ & 0 & 0 \\
\hline Pierzchajio et al, ${ }^{28} 1997$ & 24.7 (21.7- 27.9) & $0.6(0.3-1.4)$ & $91.4(89.2-93.2)$ & 0 & 0 \\
\hline Carr et al, ${ }^{29} 1998$ & $33.4(27.9-39.5)$ & $1.9(0.8-4.2)$ & $82.2(77.0-86.4)$ & 0 & 1 \\
\hline Kirby, $^{30} 2004$ & $16.8(14.0-19.9)$ & $2.3(1.4-3.8)$ & 80.1 (76.8- 83.1) & 0 & 0 \\
\hline Edwards and Norris, 2004 & $22.8(17.6-29.0)$ & $2.3(1.0-5.2)$ & 96.1 (92.4- 98.0) & 0 & 0 \\
\hline Newman et al, ${ }^{32} 2005$ & $32.5(29.2-36.0)$ & $0.9(0.5-1.9)$ & $92.7(90.6-94.4)$ & 0 & 1 \\
\hline Strayer, ${ }^{33} 2005^{b}$ & $51.3(45.1-57.3)$ & $0.8(0.3-2.6)$ & $94.6(91.1-96.7)$ & 0 & 0 \\
\hline Cotterill et al, ${ }^{34} 2005$ & $23.9(19.6-28.8)$ & $0.7(0.2-2.2)$ & $94.0(90.9-96.1)$ & 0 & 0 \\
\hline Lloyd, ${ }^{35} 2006^{b}$ & $38.0(37.2-38.8)$ & $0.5(0.46-0.54)$ & $98.4(98.2-98.6)$ & 3 & 2 \\
\hline Total & $28.9(20.4-39.3)$ & $1.7(0.9-3.0)$ & $89.2(80.1-94.4)$ & 3 & 4 \\
\hline \multicolumn{6}{|l|}{$\mathrm{Cl}=$ confidence intervals. } \\
\hline \multicolumn{6}{|c|}{ Note: rates and $95 \%$ Cls calculated using random effect model. } \\
\hline \multicolumn{6}{|c|}{ 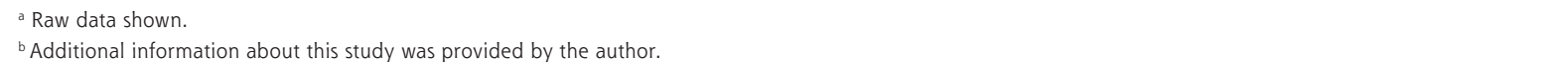 } \\
\hline
\end{tabular}

